\title{
The Patient-Physician Partnership in Asthma: Real-World Observations Associated with Clinical and Patient-Reported Outcomes
}

\author{
M. Small · A. Vickers · P. Anderson · S. Kay
}

Received: June 8, 2010 / Published online: July 30, 2010

(C) The Author(s) 2010. This article is published with open access at Springerlink.com

\section{ABSTRACT}

Introduction: It is hypothesized that a good partnership between asthma patients and their physicians has a direct and positive influence on the patients' clinical and patient-reported outcomes. Conversely, poor partnership has a detrimental effect on clinical and patientreported outcomes. This paper uses data from a real-world observational study to define partnership through matched physician and patient data and correlate the quality of partnership with observed clinical and patientreported outcomes. Methods: Data were drawn from Adelphi's Respiratory Disease Specific Programme, a cross-sectional study of consulting patients in five European countries undertaken between June and September 2009. A range of clinical and patient-reported outcomes were observed allowing analysis of the partnership between 2251 asthma patients and their physicians. Results: Analysis demonstrates that

M. Small $(\bowtie) \cdot$ A. Vickers · P. Anderson · S. Kay Adelphi Real World, Adelphi Mill, Bollington, Macclesfield, SK10 5JB, UK.

Email: mark.small@adelphigroup.com the better the partnership between patient and physician, the more likely the patient is to have their asthma condition controlled $(P<0.001)$, to experience fewer exacerbations $(P<0.001)$, to have better quality of life $(P<0.001)$, to have fewer sleep disturbances $(P<0.001)$, and to have fewer patient-reported symptoms $(P<0.001)$. Partnership is also associated with lower impact on lifestyle $(P<0.01)$ and reduced days lost at work/school $(P<0.05)$, and with patient satisfaction with their inhaler device $(P<0.05)$. Conclusion: The patient-physician partnership is a contributory factor in the improvement of asthma treatment, and patient education may lead to improvement in a patient's ability to contribute to this. Device satisfaction is one of the markers of good partnership.

Keywords: asthma; device; partnership; patient; physician; real world

\section{INTRODUCTION}

Asthma is a common inflammatory condition of the upper respiratory tract estimated to affect approximately 300 million people worldwide ${ }^{1}$ and with prevalence increasing globally by $50 \%$ every decade. ${ }^{2}$ 
Recent studies in Europe, ${ }^{3}$ the US, ${ }^{4}$ Japan, ${ }^{5}$ and the Asia-Pacific region ${ }^{6}$ indicate that asthma is underdiagnosed and undertreated and that there is considerable room for improvement in control of the condition. It has been demonstrated that optimal communication between the physician and patient is required for outcomes to be improved. ${ }^{7}$ These needs have given rise to the concept of a "patient-physician" partnership, the importance of which has been recognized across most medical communities. ${ }^{8,9}$

This paper focuses on the behavioral and attitudinal evidence collected by one researchbased organization (Adelphi, Macclesfield, UK) under the label of Disease Specific Programmes (DSPs $\left.{ }^{\circledR}\right)$. The DSPs are large, multinational, observational studies of clinical practice, designed to capture a cross-section of realworld data. These are an established method for investigating current treatment practices across a wide range of disease areas. ${ }^{10}$ The Respiratory DSP accurately reflects the current symptom prevalence and severity and associated treatment practices of respiratory conditions including asthma. ${ }^{11}$

This paper investigates the relationship between the patient and physician partnership and real-world clinical outcomes. It seeks to establish an objective assessment of the degree of partnership and compare the degree of partnership with clinical and patientreported outcomes.

\section{METHODS}

\section{Study Design}

The Respiratory DSP was conducted between June and September 2009, recruiting specialists and primary-care physicians and their patients in France, Germany, Italy, Spain, and the UK. The full methodology for this survey, including limitations of the DSP approach, has been outlined previously. ${ }^{10,11}$ Physicians completed a patient record form (PRF) for six consecutive consulting adult asthma patients, and the same patients were invited to fill out a patient selfcompletion form (PSC).

Physicians were identified by the local DSP fieldwork teams from public lists of healthcare professionals according to predefined selection criteria to ensure that the sample was representative of asthma management in each participating country. Physicians were checked for their eligibility to participate in the DSP in terms of specialty, location (hospital or office), whether they were personally responsible for treatment decisions, and how many patients they saw in a typical week (in total and with asthma in order to avoid physicians with an abnormal workload). Candidate respondents who met the predefined eligibility criteria were subsequently invited to participate in the study. To avoid potential selection bias due to variable population densities in different geographical regions in a given country, an appropriately larger sample of physicians was identified in densely populated areas than in more sparsely populated areas. As the methodology requires the next six presenting asthma patients for each physician, the DSP sample is representative of the consulting population.

All responses were anonymous to preserve patient confidentiality and to avoid bias at the data collection and analysis phases. The study protocol followed ethical procedures including informed consent of all patients for anonymous and aggregated reporting of research findings based on the questionnaires employed. Patients were instructed by the physician to complete the PSC independently and return it in a sealed envelope. Matching the physician and patient responses via patient/physician study numbers allowed the PSC data to be linked with 
comparable data recorded on the physiciancompleted PRF to highlight any areas of disparity and/or agreement. The analyses conducted for the purposes of this paper investigate data from the matched PRF and PSC records.

A number of limitations of the DSP methodology should be noted. Although respondent physicians are requested to collect data on a series of consecutive patients to avoid selection bias, in the absence of randomization, this is contingent upon the integrity of the participating respondent rather than formalized source verification procedures. Moreover, diagnosis in the target patient group is based primarily on the judgment and diagnostic skills of the respondent physician rather than on a formalized diagnostic checklist. Although the DSP can be used to identify association, a further limitation is that being cross-sectional, it cannot be used to demonstrate cause and effect.

\section{Defining Patient and Physician Partnership}

The investigators recognize that communication is a key factor in measuring the degree of partnership between the patient and the physician. The PRF and PSC records include six directly comparable questions for which the possible responses from the patient and the physician are identical and which the authors suggest provide a measure of communication and therefore partnership. These are:

- Perceived asthma severity

- Impact of condition on daily living, including sleep, getting up, daily activities, mood, relationships, leisure time, and work/ school (each assessed individually)

- Presence of the following three primary symptoms in the past 4 weeks: shortness of breath when exercising, cough, and wheezing
- Time of day of symptoms (presence of daytime and or nighttime symptoms)

- Frequency of daytime symptoms in the past 4 weeks

- Frequency of rescue inhaler usage in the past 4 weeks.

An arbitrary scoring system sensitive to the direction of the patient and physician mismatch was used to measure the level of alignment between them. An alignment score was calculated for each variable and the mean of these scores taken to produce a standardized score of between -1 and 1 for each patient. A score of zero indicates patient and physician were in perfect alignment. If a physician overstates the impact of asthma relative to a particular patient then that patient receives a positive score with maximum of 1 . If a physician understates the patient's condition relative to the patient then that record receives a negative score with minimum of -1 . The main strength of this model was that no arbitrary division of patients needed to be applied to the data and all patients could be included with an individual alignment score. Each variable of the alignment definition carried an equal weighting.

Where ordinal data were present (severity, impact of condition, frequency of daytime symptoms, and frequency of rescue inhaler usage), patients were assigned a higher score depending on the degree of mismatch. For example, on a 1-7 scale measuring the impact of asthma, every one-point difference between the patient and physician was given a score of 0.17 up to a maximum of 1 where the physician and patient rating differed by six points.

\section{Relationship Between Partnership and Clinical Outcomes}

The following clinical outcomes were analyzed against the degree of partnership between patient 
and physician: physician perception of highest achievable control, number of exacerbations in the past 12 months, EQ-5D utility score, frequency of sleep disturbances in the past 4 weeks (as measured by the Jenkins Sleep Scale), ${ }^{12}$ number of increases in symptoms not severe enough to visit the physician (as reported by patients). The following patient-reported outcomes were also analyzed: number of days lost (work/school), overall satisfaction with inhaler, and restrictions on lifestyle as a result of their asthma condition. Patients were asked to rate the restriction on their lifestyle on a five-point scale in six areas:

- What they could do in the last 12 months compared with what they used to be able to do

- How often they participate in sporting activities

- How much time they spend in sporting activities

- Social life, holidays, and leisure activities

- Activities avoided to prevent asthma worsening

- Outdoor activities during the winter or pollen season.

This resulted in a patient score between 5 (no restriction on lifestyle) and 30 (highest restriction on lifestyle).

In addition, patient age, gender, country, current/previous job, smoking status, and number of visits to any physician in the last 12 months were analyzed to determine whether these have any relationship with the patient and physician partnership.

\section{Statistical Methods}

The relationship between alignment score and each clinical outcome/variable was plotted using generalized additive models (GAM) ${ }^{13}$ to produce a smooth curve for each effect. EQ-5D utility score and age showed departures from linearity. The other variables all produced straight-line relationships once distributions had been normalized. GAMs employ sophisticated techniques that find the best additive functional form of the predictors (not necessarily linear) that model the expectation of the response variable. With flexibility in choosing functional form there is an inherent danger of overfitting, a situation in which the derived model is inaccurate for predicting responses outside of the study sample due to its complexity. To combat this, GAMs include parameter(s) that penalize the complexity of the model (often based on the second derivative of the complex function-absolute values further away from zero incurring more penalty-further away from a linear function with 0 second derivative). ${ }^{13}$

Optimal values for these penalization factors can be estimated using cross-validation techniques, where part of the sample is not used to fit the model but is only used to assess its predictive accuracy (measured as the average across $n$ replications where $n$ is the sample size-each time dropping a separate single observation, estimating parameters on the reminder, and assessing predictive accuracy on this omitted observation).

For this study, the full model, including all the fixed effects, incorporated a random variable/effect for physician in order to model the variability between physicians irrespective of their patients. This type of analysis is referred to as a Generalized Additive Mixed Model (GAMM). ${ }^{13,14}$

In terms of model specifics, nonlinear variables were modeled using penalized thin-plate regression splines (see Wood $^{13}$ for a more detailed explanation). The model was fitted using restricted maximum likelihood (REML). REML is often the preferred method over maximum likelihood (ML) because it is less likely to underestimate the variance in the model. ${ }^{15}$ This is especially true 
when the number of fixed parameters is large. ${ }^{13}$ All analysis was conducted in $\mathrm{R}$ version 2.10.0. ${ }^{16}$

\section{RESULTS}

A total of 582 physicians participated in the study (Table 1). Matched PRFs and PSCs were analyzed for a total of 2251 adult asthma patients (France 427; Germany 667; Italy 558; Spain 442; UK 157).

Figure 1 shows the outcomes and parameters that are significantly associated with patientphysician partnership (in black and grey) and those not associated with partnership (in white). The former are mainly related to severity of the disease and experience of symptoms and include: physician perception of highest achievable control for each patient; physician-recorded number of exacerbations in the last 12 months; EQ-5D utility score; patient-recorded sleep disturbances in the past 4 weeks; and the number of symptom increases in the last 12 months not severe enough to prompt a visit to the physician $(P<0.001$ for each). Parameters that impact on the patient's daily living, including restrictions on lifestyle $(P<0.01)$ and number of days lost at work or school in the last 12 months $(P<0.05)$, are also significantly associated with patient-physician partnership, as is the patient's overall satisfaction with their inhaler device $(P<0.05)$. Parameters not significantly associated with patient-physician partnership include: country, nature of employment,

Table 1. Study physicians distribution and profiles.

\begin{tabular}{lcccccc}
\hline & France & Germany & Italy & Spain & UK & Total \\
\hline PCPs & 50 & 50 & 50 & 50 & 52 & 252 \\
Specialists & 70 & 70 & 70 & 70 & 50 & 330 \\
\hline Total & 120 & 120 & 120 & 120 & 102 & 582 \\
\hline
\end{tabular}

PCPs=primary care physicians.

Figure 1. Parameters for which there is or is not a significant association with partnership between patient and physician.

Highest achievable control (physician perception)
No. of exacerbations in past 12 months (physician recorded)

EQ-5D utility score

Frequency of sleep disturbances in past 4 weeks

No. of symptom increases in past 12 months not severe enough to prompt visit to physician Restrictions on lifestyle No. of days lost at work/school in past 12 months due to asthma Overall satisfaction with inhaler

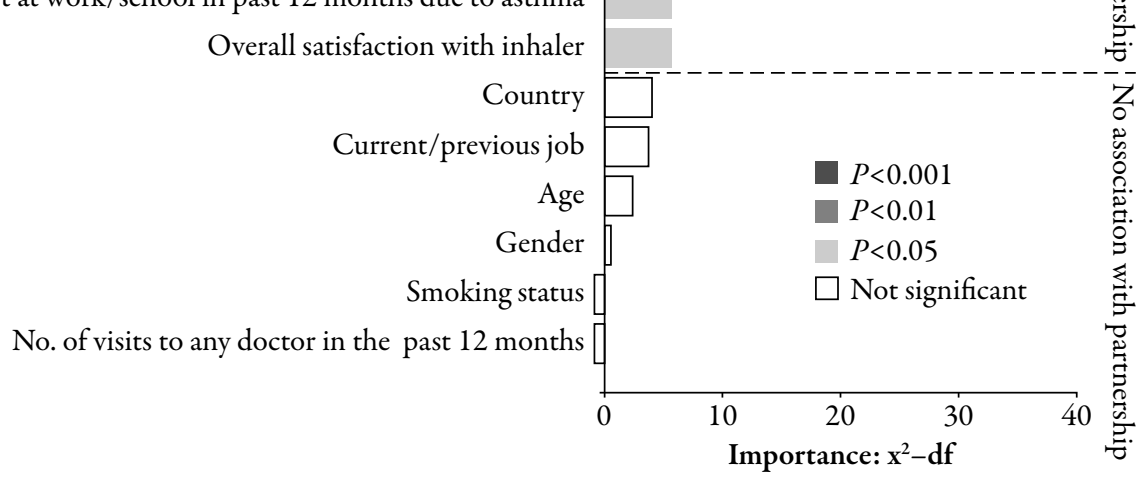


patient age, patient gender, smoking status, and number of visits to any physician in the last 12 months.

These findings are further illustrated in Figures 2-6. In Figure 2, the GAMM analysis demonstrates that better partnership between patient and physician is associated with better asthma control (as perceived by the physician). Patients whose asthma is controlled have a mean alignment difference of 0.023 compared with 0.03 among patients whose asthma is not controlled.

Similarly, in Figures 3-6, better partnership is associated with fewer exacerbations, better quality of life (as measured by EQ-5D utility score), fewer sleep disturbances (as measured by Jenkins' sleep scale), and fewer patientreported symptoms. In each case, as the degree of alignment increases, the clinical outcome is better. This pattern is also observed when GAMM analysis is applied to impact on daily living (Figures 7-8), where as alignment increases, patients are more likely to report lower restriction on their lifestyle and reduced days lost at work/school.

Finally, it was observed that patient satisfaction with device was significantly

Figure 2. Better patient-physician partnership is associated with control of asthma (as perceived by the physician; $P<0.001)$.

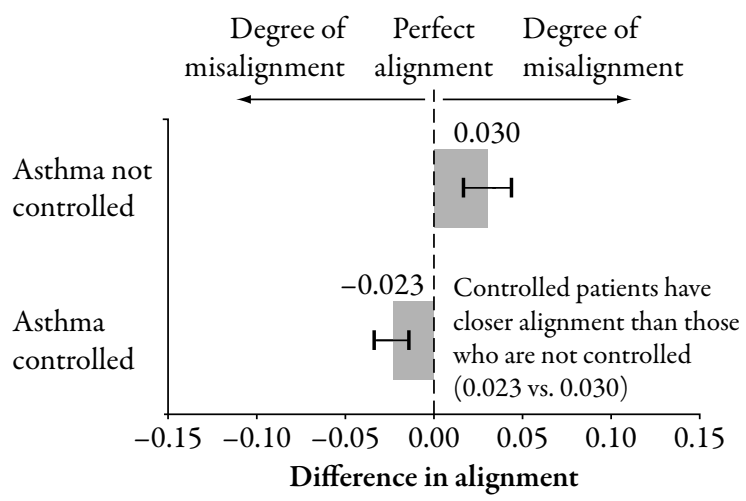

Figure 3. Better patient-physician partnership is associated with lower number of exacerbations in past 12 months (as recorded by the physician; $P<0.001$ ). Shading represents one standard error.

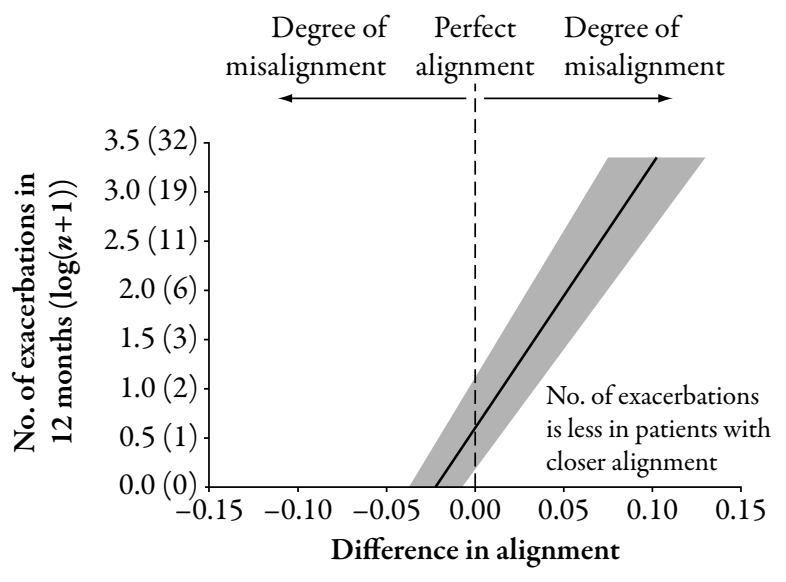

Figure 4. Better patient-physician partnership is associated with improved EQ-5D utility score $(P<0.001)$. $\mathrm{QoL}=$ quality of life. Shading represents one standard error.

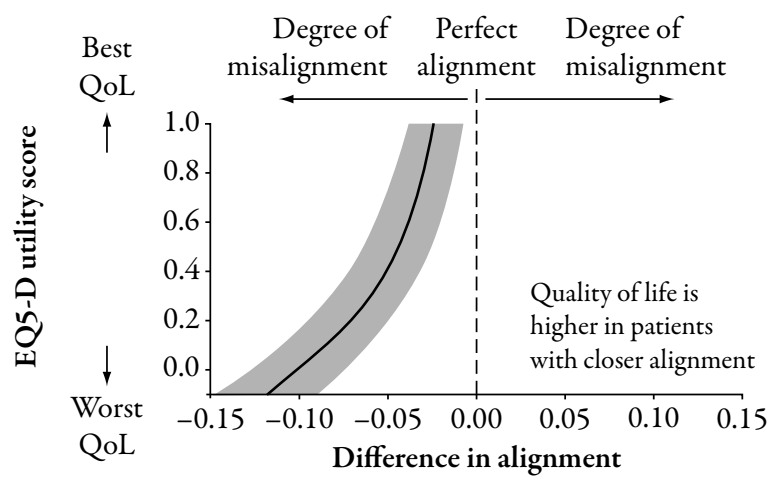

Figure 5. Better patient-physician partnership is associated with fewer sleep disturbances in the past 4 weeks $(P<0.001)$. Shading represents one standard error.

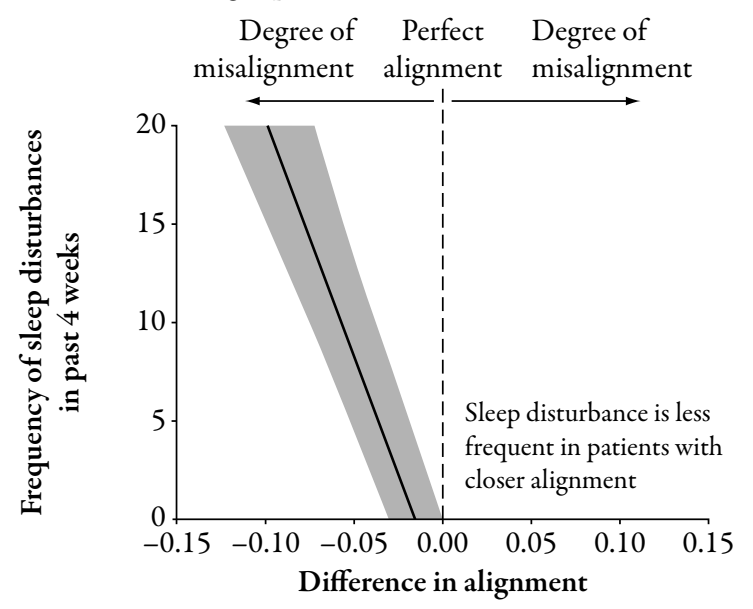


Figure 6. Better patient-physician partnership is associated with fewer symptom increases not severe enough to consult physician (as reported by patient; $P<0.001$ ). Shading represents one standard error.

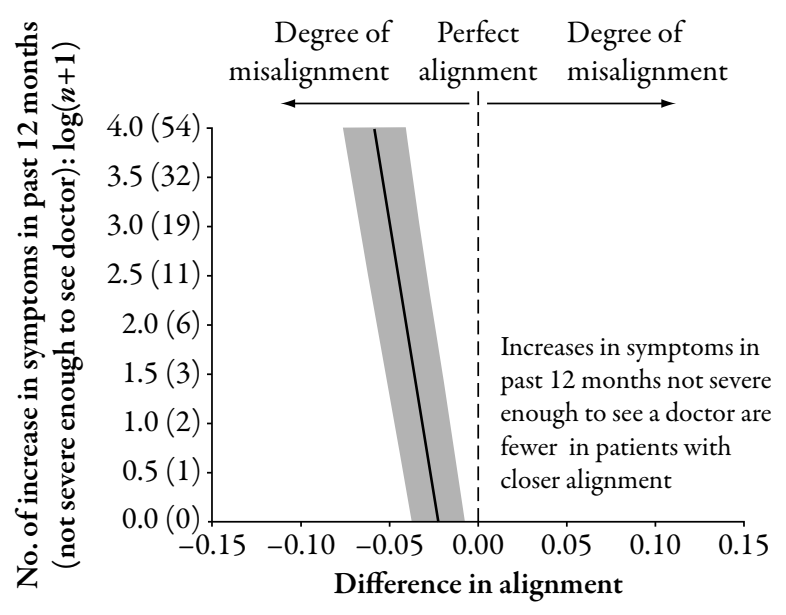

Figure 7. Better patient-physician partnership is associated with less restriction on lifestyle $(P<0.01)$. Shading represents one standard error.

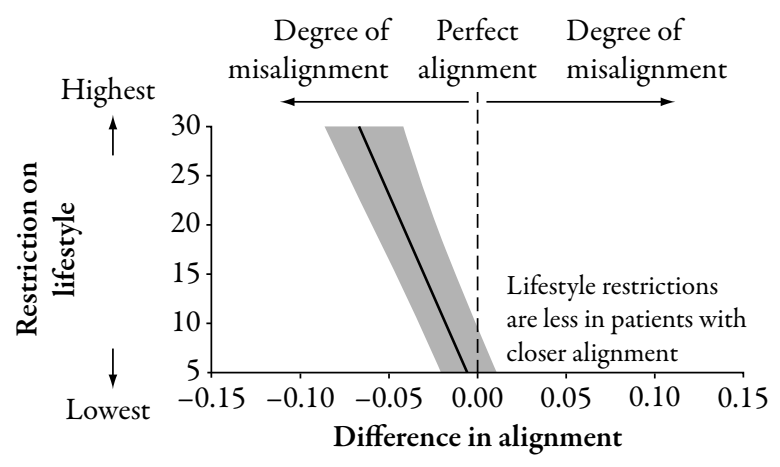

Figure 8. Better patient-physician partnership is associated with fewer days lost at work/school in past 12 months due to asthma $(P<0.05)$. Shading represents one standard error.

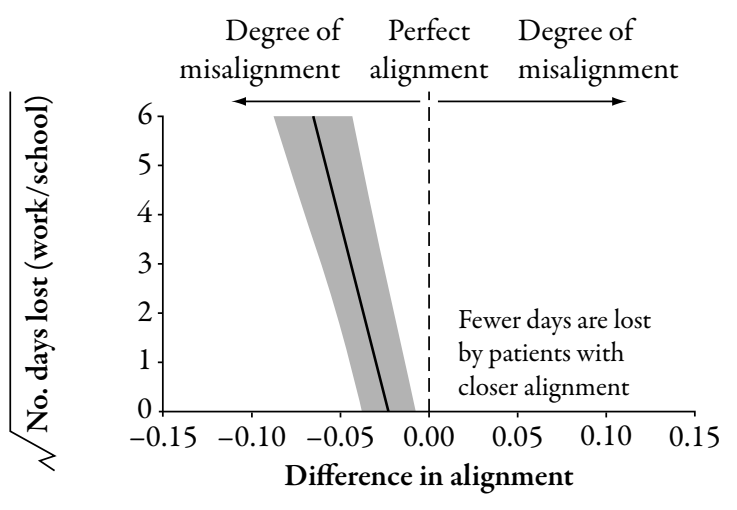

associated with partnership $(P<0.05)$ to the extent that patients who are more satisfied with their inhaler are more likely to have good partnership with their physician than those who are dissatisfied with their device.

\section{DISCUSSION}

The importance of partnership between the asthmatic patient and their physician is widely recognized by the medical community. The aim of the present study was to provide evidence from real-world observational data to objectively assess the impact on clinical and patient-reported outcomes for asthma patients with good "partnership."

The DSP collects data from patients and physicians, and several important clinical and disease diagnosis data points can be used to investigate the same subject/clinical assessment. A good partnership is a strong alignment between patient and physician on these variables, for which it is argued that a high degree of alignment can only be achieved if good communication exists between the patient and the physician.

A number of clinical criteria were selected that accurately record the status of disease, the confirmed clinical assessment, improvement or decline in disease, and well-being of the patient. These were measured for each pairing of patient and physician and compared with the quality of partnership in each pairing.

It might be expected that parameters such as the patient's age, gender, occupation, smoking status, and number of visits to the doctor would have an association with the degree of patientphysician partnership in terms of time and quality of patient and physician interactions, but this does not appear to be the case. Further work is needed to understand the role of these patient characteristics. 
Despite the limitations of the DSP approach acknowledged in the methodology, the study has demonstrated an association between good partnership and asthma disease outcome for the patient. Undoubtedly, as reported in the literature, physician-patient communication is an aspect that influences the depth of partnership and similarly the widely reported need to improve the level of patient disease awareness and education will be influential in the quality of communication between physician and patient.

The inhaler device and patient satisfaction with the device is one marker of a good partnership, which in turn correlates with a more positive outcome of the patient. It is suggested that assessing patient satisfaction with device can be a swift observation for determining quality of partnership and providing the basis for a relationship between physician and patient that fosters an opportunity for improved clinical outcomes. It would be valuable to investigate this finding further, perhaps through a longitudinal study including an assessment of the benefits of physician education to improve patient-physician partnership and associated patient outcomes.

In conclusion, partnership is dependent on good communication between the patient and the physician. ${ }^{8,9}$ A key measure of good communication is alignment between the patient and physician on the impact of asthma. This study demonstrates that good alignment on these factors is associated with better outcome for the patient. It was observed that satisfaction with choice of device is an important marker for partnership between the physician and the patient. One possible way to encourage partnership would be to invest in patient education to improve patients' ability to contribute. Further work is required to understand and to promote improvement in alignment between the physician and patient.

\section{ACKNOWLEDGMENTS}

This study was supported by AstraZeneca. All listed authors meet the criteria for authorship set forth by the International Committee for Medical Journal Editors.

Open Access. This article is distributed under the terms of the Creative Commons Attribution Noncommercial License which permits any noncommercial use, distribution, and reproduction in any medium, provided the original author(s) and source are credited.

\section{REFERENCES}

1. Braman S. The global burden of asthma. Chest. 2006;130:4S-12S.

2. Masoli M, Fabian D, Holt S, et al. Global Initiative for Asthma (GINA) program: the global burden of asthma: executive summary of the GINA Dissemination Committee report. Allergy. 2004;59:469-478.

3. Rabe KF, Vermeire PA, Soriano JB, et al. Clinical management of asthma in 1999: the Asthma Insight and Reality in Europe (AIRE) study. Eur Respir J. 2000;16:802-807.

4. GlaxoSmithKline. Asthma in America: a Landmark Survey. 1998. Available at: http://www. asthmainamerica.com. Accessed March 25, 2010.

5. Adachi M, Morikawa A, Ishihara K. Asthma insights and reality in Japan (AIRJ). Arerugi. 2002;51:411420. Article in Japanese.

6. Lai CK, De Guia TS, Kim YY, et al. Asthma control in the Asia-Pacific region: the Asthma Insights and Reality in Asia-Pacific Study. J Allergy Clin Immunol. 2003;111:263-268.

7. Volovitz B, Friedman HN, Levin S, et al. Increasing asthma awareness among physicians: impact on patient management and satisfaction. J Asthma. 2003;40:901-908.

8. Saba GW, Wong ST, Schillinger D, et al. Shared decision making and the experience of partnership in primary care. Ann Fam Med. 2006;4:54-62.

9. Clark NM, Nohwehr F, Gong M, et al. Physicianpatient partnership in managing chronic illness. Acad Med. 1995;70:957-959. 
10. Anderson P, Benford M, Harris N, et al. Realworld physician and patient behaviour across countries: Disease-Specific Programmes - a means to understand. Curr Med Res Opin. 2008;24:30633072 .

11. Higgins V, Kay S, Small M. Physician and patient survey of allergic rhinitis: methodology. Allergy. 2007;62:6-8.

12. Jenkins CD, Stanton BA, Niemcryk SJ, Rose RM. A scale for the estimation of sleep problems in clinical research. J Clin Epidemiol. 1988;41:313-321.
13. Wood SN. Generalized Additive Models: an Introduction with R. Press, Boca Raton, FL: Chapman and Hall/CRC Press; 2006.

14. Wood SN. 2009. Gamm: generalized additive mixed models. Available at: http://CRAN.R-project. org $/$ package $=$ mgcv. Accessed July 12, 2010.

15. Pinheiro JC, Bates DM. Mixed-Effects Models in S and S-PLUS. New York: Springer-Verlag; 2000.

16. R Development Core Team. 2009. R: a Language and Environment for Statistical Computing. Vienna: R Foundation for Statistical Computing. Available at: http://www.R-project.org. Accessed July 12, 2010. 